\title{
The Research Experience for Undergraduates (REU) Principal Investigators (PI) Guide: Development of a Best Practices Website
}

\section{Ms. Mariangely Iglesias Pena, Iowa State University}

Mariangely Iglesias Pena is an MS student in Human Computer Interaction at Iowa State University's Virtual Reality Applications Center. Her background is in industrial design, which drives her interest in interactive and web design.

\section{Prof. Stephen B. Gilbert, Iowa State University}

Stephen B. Gilbert received a BSE from Princeton in 1992 and $\mathrm{PhD}$ from MIT in 1997. He has worked in commercial software development and run his own company. He is currently an assistant professor in the Industrial and Manufacturing Systems Engineering department at Iowa State University, as well as Associate Director of ISU's Virtual Reality Application Center and its Graduate Program in Human Computer Interaction. His research focuses on technology to advance cognition, including interface design, intelligent tutoring systems, and cognitive engineering.

\section{Jamie Payton, Temple University}

Dr. Jamie Payton is an Associate Professor of Computer and Information Sciences at Temple University. She received her Ph.D. in Computer Science at Washington University in St. Louis in 2006. Her research interests include crowdsensing, smart and connected health, and software engineering for pervasive computing environments. Dr. Payton is also committed to broadening participation in computing and advancing computer science education. She is the Director of the STARS Computing Corps, an NSFfunded alliance of 53 colleges and universities that aims to broaden participation in computing and the co-founder of the International Conference for Research on Equity and Sustained Participation in Engineering, Computing, and Technology (RESPECT). 


\title{
The REU Principal Investigator's Guide: Development of a best practices website
}

\begin{abstract}
With the help of the National Science Foundation (NSF), many Principal Investigators (PIs) have been able to mentor undergraduates through Research Experience for Undergraduates (REU) site awards. These REU sites are critical to the development of future graduate students, but can be challenging to run due to several required skills outside the scope of most faculty members' expertise, e.g., recruiting applicants, navigating the logistics of housing visiting undergraduate students, and tracking student outcomes after their REU experiences. In recent years, REU PIs in NSF's Computer \& Information Science \& Engineering (CISE) Directorate have come together through PI meetings to share best practices for running a successful REU site. While PIs inevitably take different approaches to running their sites based on their research projects, there is still a need to provide new PIs with guidance on the different aspects of an REU site such as identifying resources that can assist in recruiting women and underrepresented minority applicants, providing training for graduate students acting as mentors, and strategies for keeping a mentoring connection to undergraduate researchers after they return to their home institutions.

Currently, REU site preparation and orientation for new PIs is a face-to-face process that requires careful planning and significant travel costs. The REU PI Guide, a set of web-based resources at https://www.vrac.iastate.edu/cise-reu-pi-resources/, was developed to share best practices of experienced PIs and build capacity within the REU PI community in a more scalable and cost-effective way. The REU PI Guide allows PIs to look up advice and guidance when needed and share their own best practices. This paper describes our approach to designing the REU PI Guide. The Guide is a database of documents, examples, and overviews of the different aspects of running an REU site. The Guide was developed by assessing new PIs' needs at an NSF workshop for new PIs, gathering existing resources from experienced PIs, creating and refining a website, and evaluation with new PIs. The website's content and design will be refined through on-going feedback from PIs and other REU site stakeholders. This site has the potential broader impact to share best practices with REU PIs outside the CISE directorate and significantly ease the process of engaging future scientists via REU sites.
\end{abstract}

Introduction

The CISE directorate of NSF has funded Research Experience for Undergraduates (REU) sites for over 30 years, offering hundreds of faculty principal investigators (PIs) the experience of mentoring undergraduates through research projects by running an REU site. Starting in 2002, CISE has organized REU PI meetings to share best practices among PIs to raise the quality of implementation across all sites. According to Matzen and Alrifai [1], however, a 2008 SIGCSE survey showed notable differences in PIs' objectives for and conceptualization of undergraduate research. Thus, while the structure of successful REU sites can vary, there is still a need for consistent guidance on the aspects of administering an REU site with which typical PIs have less experience. Because computer science PIs are less familiar with program evaluation methods, for example, Rorrer [2] began scaffolding consistent evaluation practices within CISE REU sites in 2009 using her A La Carte Survey and CISE REU Toolkit. 
This paper describes a research effort to raise the overall quality of site implementation by creating the REU PI Guide, a set of web-based resources developed to help new REU site PIs prepare and run an effective REU site efficiently. According to user-centered design principles $[3,4]$, the REU PI Guide will be more effective if the needs of all the stakeholders are considered. The primary stakeholder is the REU site PI. But there are multiple secondary stakeholders whose needs the PI must consider, including: program coordinators who may assist the PI, faculty project mentors, graduate student mentors, the REU interns themselves, and NSF program directors. The REU PI Guide design effort focused on providing PIs with resources needed to satisfy the needs of all stakeholders satisfactorily.

\section{Goals of the REU PI Guide}

A primary goal of the REU PI Guide was to significantly smooth the process of running REU sites for new PIs. Sometimes due to federal budget delays, PIs receive their new REU site award with very short notice, and the first REU summer may be organized with a more ad hoc approach than desired. Ideally the REU PI Guide would help avoid this situation and yield a better result for all stakeholders. A secondary goal was the Guide provide material that is useful to REU PIs outside the CISE directorate.

The REU PI Guide would ideally act as a database to keep documents, examples, and overviews of the different aspects of running an REU site PIs to reference and use as they prepare for the upcoming REU. In addition, if these documents and templates come from experienced PIs to be shared with new PIs, there is the possibility that the Guide would aid in building an REU PI community of practice [5].

\section{Previous Work}

While significant research is published about REU sites, especially in education research conferences such as SIGCSE (computer science education), ASEE (engineering education), and other discipline-specific conferences, many of these papers focus on summarizing best practices or lessons learned from the faculty perspective for performing research with undergraduates [68]. Some of them focus more on the structure of the REU site, but few of them span the breadth of multiple REU sites, attempting to synthesize across sites. Also, while the authors have seen websites that attempt to gather resources for REU PIs, e.g., http://bioreu.org/resources and http://cisereu.org/, to our knowledge, these have not undergone a systematic design and evaluation process.

Methods

Per Steve Krug's famous web design guide, Don't Make Me Think [9] and the more academic model of usability by Don Norman [10], the design of any system should match the expectations of the user. Using this user-centered design approach, six steps were completed. Details follow.

1. A protocol for gathering human subjects data was approved by the local institutional review board (IRB).

2. User requirements were gathered at a new PI workshop of 26 new PIs in March, 2017. 
3. Interviews of six experienced CISE REU PIs were conducted (two of the six were authors on this paper) to get a sense of their best practices.

4. Based on interviews, new PI needs, and published literature from SIGCSE and ASEE, materials for the REU PI Guide were gathered.

5. The REU PI Guide website was created.

6. The REU PI Guide was evaluated by 22 REU PIs, the subset of the 737 REU PIs who were contacted with an evaluation survey.

Results

In preparation for the initial workshop for the new PIs, a registration form was sent asking about concerns or major questions they had about running an REU site. Figure 1 shows a histogram of their concerns. For the purpose of this project, the top two concerns were chosen as the initial focus of the REU PI Guide, given magnitude of information both of those topics require.

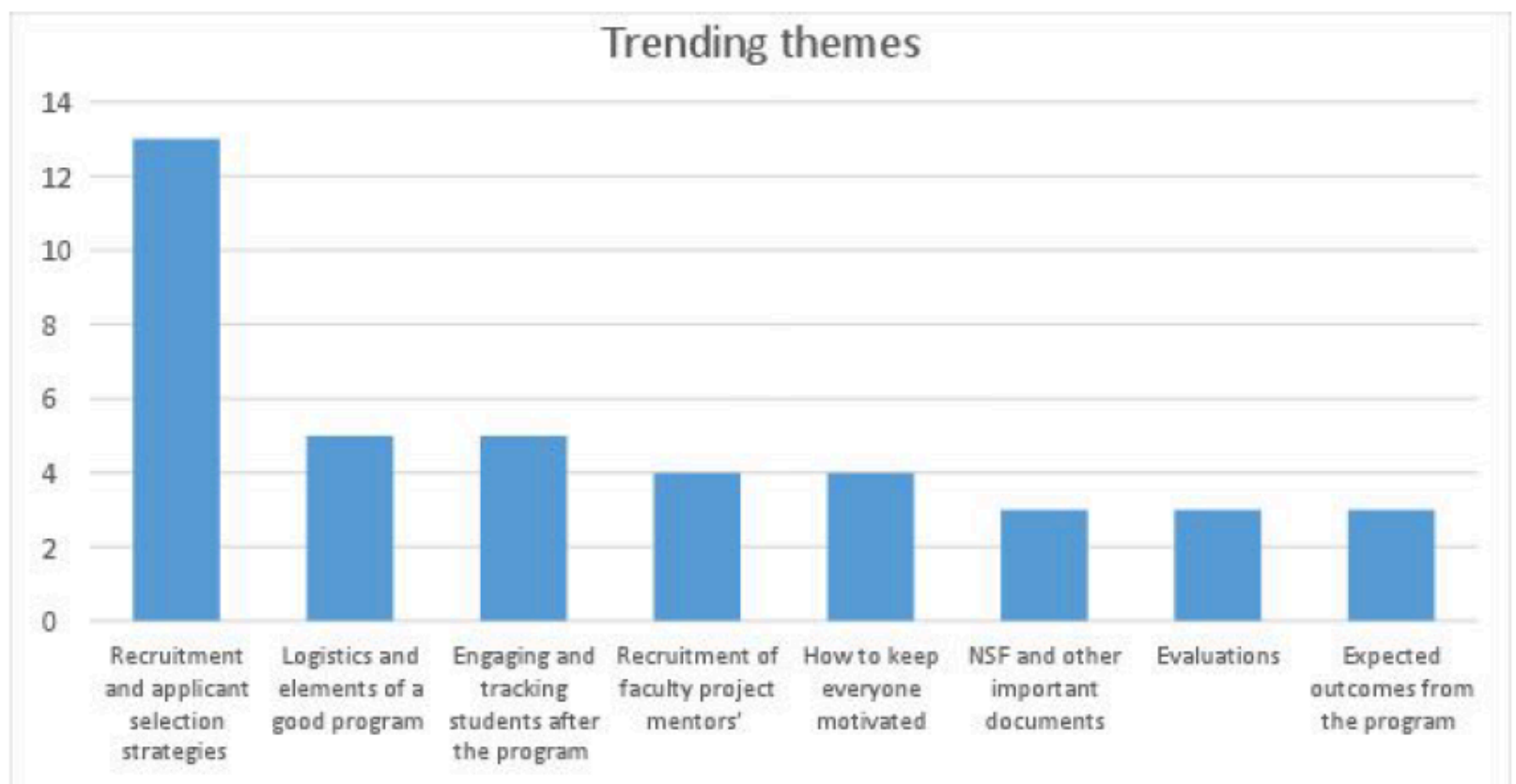

Figure 1: Themes of the questions noted from 26 participants of the new CISE REU PI workshop

The biggest identified problem was the lack of a common, centralized, source of information where PIs can go to and search for guidance and resources to use when planning of running their site. Meaning that by providing a place they can go to, they can gather information in a quicker and reliable way rather than worrying what to do next. The workshop and interview outcomes led to the following design requirements for the content of the REU PI Guide:

The REU PI Guide website must contain the following:

1. Information presented by topic

2. Downloadable templates and resources for PIs to refer to or customize

3. Identified best practices within larger topics.

a. Larger or dense topics should have identified pro-tips based on experienced PIs' wisdom 
Also based on the workshop and interviews, user needs in terms of $\boldsymbol{u s a b i l i t y}$ were established. The user of the REU PI Guide should be able to:

4. Navigate the website by topic

5. Identify the type of file of any downloadable resource

6. Download provided resources

7. Identify pro-tips

This combination of requirements set up the following design space tradeoffs for the Guide: 1) High cognitive load vs. amount of information and 2) Topic- vs. Task-based organization. Since running an REU is a year-long process and contains many moving parts, it is important to consider the most important pieces of information that should be provided. On one hand, providing all (or more of) the necessary information can help explain in detail all the moving parts, but it might become overwhelming for the user to read. This is especially true if the information is presented as full paragraphs or written as literary or written work. Hence, it's important to create an information architecture that balances the provided information and resources, the degree and manner in which it expose the information. The information structure can benefit from proper labels, hierarchy, and chunking content which can help minimize cognitive load [11].

Topic-based organization is often taken as an approach to organize content, especially the top tap menu in an app, in terms of topics, similar to what newspapers, academic departments, and nonfiction books do [12]. However, this approach can lead to limited breath of the provided content, which in this case could limit the ability to incorporate more information if demanded by the REU PIs. Task-oriented schemes often organize a website by the expected tasks or processes a user will want to perform [12]. Although task-oriented is considered to be more usercentered, it's rare to find a website that is solely design as task-oriented, due the different tasks a user might perform and the potential challenge to the flow and structure of the website. The best approach is to identify a task/topic hybrid scheme which allows information flexibility and allows for a stronger user-centered approach. During the workshop, we had the PIs do a card sorting activity by different topics [13], which allows us to identify the different tasks, questions, and sub-topics that needed to be addressed under a major category.

The design of the website was supported by affinity diagramming, task analysis, and other leading techniques of user-centered design $[13,14]$. The results from these techniques guided the design of the information architecture. Once the Guide was drafted (it can be seen at https://www.vrac.iastate.edu/cise-reu-pi-resources/), an evaluation was conducted using a Qualtrics survey. The following three tasks were chosen based on the topics of importance shown in Figure 1.

a. Task 1: Go to the website and find information about accepting/rejecting applications. Are there downloadable templates or examples, and do they open? Do they provide the expected information?

b. Task 2: Go to the website and find information about what you need to do after accepting your students and preparing your REU site before your students' arrival (e.g., getting them to the REU site, accommodations, etc.). Are there downloadable templates or examples, and do they open? Do they provide the expected information? 
c. Task 3: Go to the website and find information about student tracking after the program. Are there downloadable templates or examples, and do they open? Do they provide the expected information?

Participants were recruited from the online NSF list of REU sites, and thus included CISE and non-CISE PIs. After consenting to participate, each participant browsed the REU PI Guide website and attempted to complete these tasks. After completion, participants continued to an evaluation survey. The evaluation consisted in using the USE (Usefulness, Satisfaction, and Ease of Use) questionnaire, a type of qualitative questionnaire that has been used by various companies to determine user satisfaction and frequency of use [15]. The questionnaire is broken down into 4 parts (Usefulness, Satisfaction, Ease of learning, and Ease of Use), all partaking in a 5-point Likert-scale (strongly disagree, somewhat disagree, neither agree nor disagree, somewhat agree, and strongly agree) including a "n/a" for users who feel like a specific question does not apply to them or their experience.

Overall, results were very positive, with an average of 78\% of all Likert responses being "somewhat agree" or "strongly agree." Participants were also asked for any negative or positive comments they had about the REU PI Guide. Results are shown in the table below. The overall survey response rate was 3\%, so there is potential for bias in reported results. Getting REU PIs to complete the surveys is an on-going challenge.

\begin{tabular}{|c|c|}
\hline Negative Aspects & Positive Aspects \\
\hline 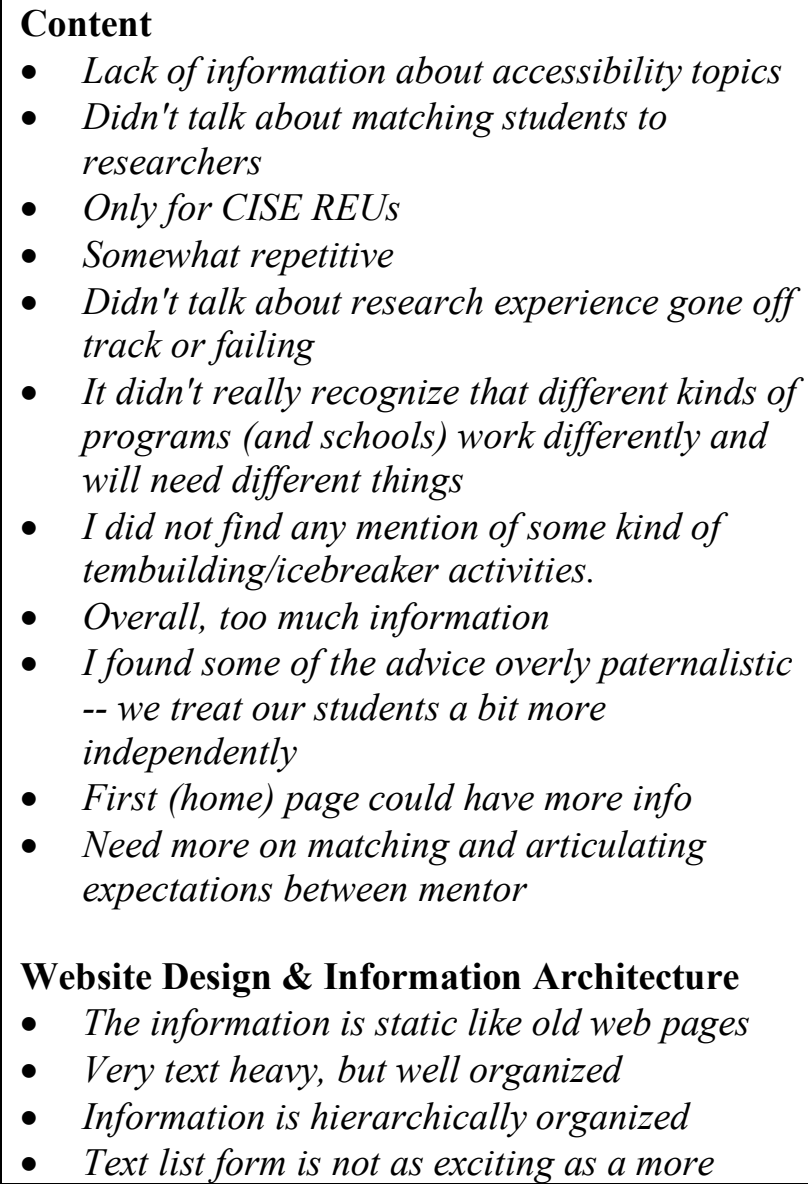 & 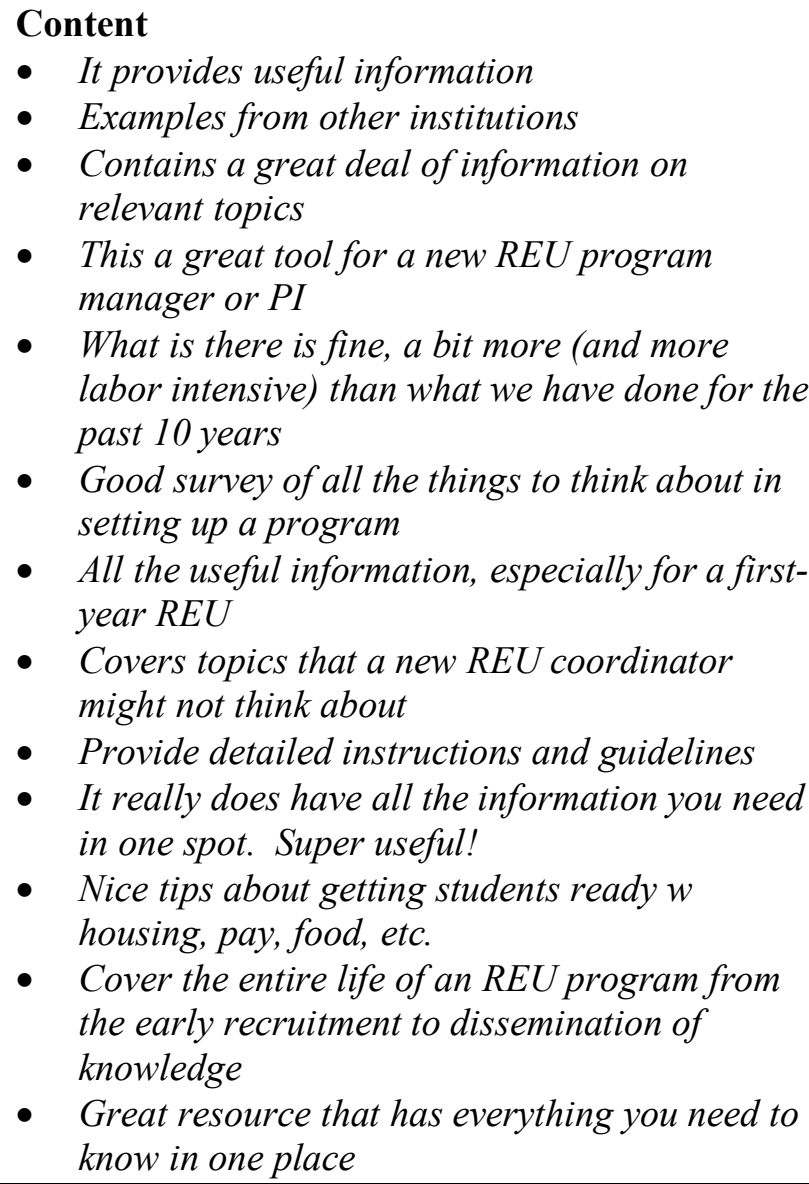 \\
\hline
\end{tabular}




\begin{tabular}{|l|l|}
\hline $\begin{array}{l}\text { visual interaction/tool } \\
\text { Organizing both by time and by topic (now } \\
\text { links are by topic) would be nice, like what do I } \\
\text { need to know before, first week, ongoing and } \\
\text { after the program }\end{array}$ & $\bullet \begin{array}{l}\text { Pro tips (noted three times) } \\
\text { came from experienced people }\end{array}$ \\
- Lack of images & - Has the appropriate information \\
- Too much text to read through & Website Design \& Information Architecture \\
- It is boring! & $\bullet \quad$ Calendar was very useful (noted four times) \\
& $\bullet \quad$ Very well-organized website \\
\hline
\end{tabular}

Discussion \& Implications for REU PIs in the Future

The list of negatives aspects mentions how there is a lack of awareness of the breadth of style of REU sites, particularly beyond the CISE discipline. This critique can be expected, since CISE was the initial focus. However, filterable versions of the Guide could be created in the future, in which the user chooses a discipline, and irrelevant resources are hidden. There are some discrepancies between users about amount of desired information, with some wanting more and others claiming there was too much already. Addressing this issue would require further investigation via interviews with our target audience. We can also see some concerns around the website's template (static), heavy text, and information architecture. These can also be improved in a future version.

The list of positive aspects mentions how users appreciated or agreed with the content provided in the website. They thought a lot of the information was relevant, contained great examples and tips, complete and detailed, and useful. There was some mention of how well the website was organized, and the ability easily to identify "pro-tips." The timeline and various examples/resources were well received and appreciated. Additionally, there were mentions on how easy, clear, and simple the website was to use.

Based on the evaluation's data, the authors suggest that while not perfect, the first iteration of the CISE REU PI Guide was satisfactory, useful, and welcomed by the participants. The provided information matched their expectations, and left them requesting more materials or specific guidance. This means that there can be an opportunity to either build a more community-based website where PIs can request or provide more guidance based on their needs, and building a non-CISE-specific REU website for new (or all) PIs which they can use as a guide or checklist during their planning.

In general, the use of this type of prototype and evaluation process did open up the opportunity to get a lot of feedback and participants to evaluate the system. Participants were not shy in voicing their concerns, favorite parts, and things that needed to be addressed, especially since they could explore and answer questions on their own time. Overall, it was a useful approach for this type of exercise and being able to gather a lot of participants from the USA, but the big variance in our participants' disciplines might not give us an entirely clear picture of whether the information and style of website fits our users. Therefore, the best approach would be to gather a smaller and more focused group of participants (our main users) and do smaller and supervised sessions, which would allow to ask follow up questions and fully understand what needs to be changed, how, and the reason why. 
References

[1] R. Matzen and R. Alrifai, "Defining undergraduate research in computer science: a survey of computer science faculty," Journal of Computing Sciences in Colleges, vol. 27, no. 3, pp. 31-37, 2012.

[2] A. S. Rorrer, "An evaluation capacity building toolkit for principal investigators of undergraduate research experiences: A demonstration of transforming theory into practice," Evaluation and program planning, vol. 55, pp. 103-111, 2016.

[3] J. J. Garrett, Elements of user experience, the: user-centered design for the web and beyond. Pearson Education, 2010.

[4] J. Gothelf and J. Seiden, Lean UX: Applying lean principles to improve user experience. " O'Reilly Media, Inc.", 2013.

[5] E. Wenger, Communities of practice: Learning, meaning, and identity. Cambridge university press, 1998.

[6] K. Ward, "Research with undergraduates: a survey of best practices," Journal of Computing Sciences in Colleges, vol. 21, no. 1, pp. 169-176, 2005.

[7] P. Stephenson, L. Miguel, J. Peckham, J.-Y. Herv, \#233, and R. Hutt, "Using undergraduate interdisciplinary research to promote computer science," J. Comput. Sci. Coll., vol. 22, no. 3, pp. 98-104, 2007.

[8] B. K. Dean and O. A. Rawashdeh, "An Interdisciplinary Undergraduate Research Experience Program in Electrical and Computer Engineering-Lessons Learned through 6 Years of Program Operations," in 2017 ASEE Annual Conference \& Exposition, 2017.

[9] S. Krug, Don't make me think: A common sense approach to web usability. Pearson Education India, 2005.

[10] D. Norman, The design of everyday things. New York: Basic Books, 1988.

[11] S. Feinberg and M. Murphy, "Applying cognitive load theory to the design of web-based instruction," in Proceedings of IEEE professional communication society international professional communication conference and Proceedings of the 18th annual ACM international conference on Computer documentation: technology \& teamwork, 2000, pp. 353-360: IEEE Educational Activities Department.

[12] L. Rosenfeld, P. Morville, and J. Arango, Information architecture for the web and beyond, 4th ed. O'Reilly Media, 2015.

[13] H. Beyer and K. Holtzblatt, "Contextual design," interactions, vol. 6, no. 1, pp. 32-42, 1999.

[14] W. Albert and T. Tullis, Measuring the user experience: collecting, analyzing, and presenting usability metrics. Boston: Morgan Kaufmann, 2013.

[15] A. M. Lund, "Measuring usability with the use questionnaire12," Usability interface, vol. 8, no. 2, pp. 3-6, 2001. 